\title{
Towards Damage Controlled Hot Forming
}

\section{BAMBACH Markus ${ }^{1, a^{*}}$, SIZOVA Irina ${ }^{1, b}$ and EMDADI Aliakbar ${ }^{1, c}$}

\author{
${ }^{1}$ Brandenburg University of Technology (BTU), Chair for Mechanical Design and Manufacturing, \\ Konrad-Wachsmann-Allee 17, 03046 Cottbus, Germany
}

abambach@b-tu.de, bsizova@b-tu.de, ’emdadi@b-tu.de

Keywords: metal forming, damage control, titanium aluminides, isothermal forging.

\begin{abstract}
Metal forming processes may induce internal damage in the form of voids in the workpiece under unfavorable deformation conditions. Controlling the amount of damage induced by metal forming operations may increase service performance of the produced parts. Damage is crucial in high-performance components of limited workability such as jet engine turbine blades. Recent developments have introduced forged titanium aluminides into commercial jet engines. Titanium aluminides are lightweight intermetallic compounds with excellent creep properties but very limited ductility. Their low workability requires isothermal forging at slow strain rates, which is typically kept constant in the process. This work explores the possibility of increasing the ram speed during the process so that the process time is reduced while the amount of damage introduced into the workpiece is controlled. The results show that a $25 \%$ reduction in process time seems viable without increase in damage by solving an optimal control problem, in which the ram speed profile is determined off-line by minimization.
\end{abstract}

\section{Introduction}

In the production of semi-finished products, hot forming is typically used to remove defects such as pores and voids from the casting process and to adjust the microstructure for further processing. Although pores and voids can be closed during hot forming under compressive stresses, hot working processes can also promote damage. The initiation of damage during hot forming is determined by the local stresses at grain boundaries and interfaces. In contrast to cold forming, thermally activated softening processes (recovery and recrystallization) occur during hot forming. These thermally activated softening processes can be used to control damage in hot forming processes, which would allow for damage-controlled processing. Damage control is of great relevance for materials with low workability such as intermetallic compounds. Titanium aluminides (TiAl) of the second and third generation have been introduced recently as forged blades into civil aircraft in two- or three-shaft engines. The use of TiAl alloys with a density of approx. $3.9 \mathrm{~g} / \mathrm{cm}^{3}$ pursues the goal of replacing the heavy nickel-based superalloys currently used in the hightemperature range, thus achieving a drastic weight reduction. Forged blades are generally considered superior to cast blades [1]. Especially for highly loaded turbine components with very high rotational speeds such as the 'geared turbofan' [2], the necessary properties can only be achieved via a complex process chain, which includes isothermal die-forging as propertydetermining process.

TiAl blades are currently forged in two stages in the industry. First, the volume distribution necessary for the finish forging is achieved by lateral extrusion of a cylindrical preform, then the final forging is performed [3]. Due to the highly anisotropic and inhomogeneous deformation of two- and three-phase TiAl alloys, the forming is carried out in a narrow process window. In terms of temperature, the process window is limited towards lower temperatures by the disordering temperature of the phases and the mechanical strength of the dies. Towards higher temperatures, both the two-phase area $\alpha+\beta$ and the thermal load capacity of the dies set a limit. This results in a temperature window of $\sim 100 \mathrm{~K}$. Since the microstructure development depends strongly on the temperature, all forming processes are carried out almost isothermally. For the common alloys of the third generation, dies are heated to temperatures of $\sim 1200{ }^{\circ} \mathrm{C}$ and the billets to tempera tures 
between 1200 and $1350{ }^{\circ} \mathrm{C}$. However, the high process temperatures are not sufficient to shape the material at higher strain rates [4,5]. As forging tool material, an expensive particle-reinforced molybdenum-hafnium-carbon alloy (MHC) is typically used.

Despite the success achieved with isothermal forging, sustainability and cost issues persist. By reducing component costs, forged TiAl components could not only be increasingly used in aircraft turbines, but also in other applications, e.g. for turbines of helicopters. Previous work by the authors of this paper put forward a new process design, which is based on pre-assembled die sets [6]. For smaller components, this process design allows for a cost advantage by reducing the idle time of the forging press. Another possibility to increase the rate of produced parts is to accelerate the isothermal forging process. So far, isothermal forging processes are conducted with a constant, very slow strain rate. The forging process of a turbine blade currently takes $\sim 4-5$ minutes for the production of the preform and for the final-forging each (cf. [7]). TiAl materials initially show a steep increase in yield stress during hot forming, but even at low degrees of deformation they reach a pronounced yield stress maximum and then show a steep yield stress drop. So far, the strong softening is not used to accelerate forming processes after passing through the maximum load. If the softening in the workpiece could be used to accelerate the forming process, the process times could be reduced significantly. This paper investigates the possibility to accelerate the isothermal forging process by optimizing the ram speed profile. Since the forging of a turbine blade yields locally different degrees of deformation, an optimal control problem must be solved.

\section{Materials and Methods}

The section 'materials and methods' gives an overview of the titanium aluminide alloy used in this study, the testing procedure employed to analyze the strain hardening behavior and the models used to analyze a cylindrical upsetting problem, which is employed to investigate the optimal control problem. The formulation of the optimal control problem and the solution prodecure are also detailed in this section.

Material preparation. The material used in this study is a titanium aluminide alloy commercially known as TNB-V5 with a nominal composition $\mathrm{Ti}-45 \mathrm{Al}-5 \mathrm{Nb}-0.2 \mathrm{~B}-0.2 \mathrm{C}$ (at.\%). Isothermal compression tests were performed at temperatures of $1150-1250{ }^{\circ} \mathrm{C}$ and at strain rates of $\dot{\varepsilon}=0.001-$ $0.1 \mathrm{~s}^{-1}$ using a deformation dilatometer. Gas-atomized powder with an average particle size of about $45 \mu \mathrm{m}$ was consolidated by means of the field-assisted sintering technique (FAST), also known as spark plasma sintering (SPS), using an HP D10-SD machine (FCT Systeme GmbH, Germany). The sintering process was performed using graphite tools with a diameter of $40 \mathrm{~mm}$. The graphite tool unit was filled with the powder. For technological reasons, a graphite film was placed between the powder and the die and punches. The sintering process was conducted in vacuum at a sintering temperature of $1250{ }^{\circ} \mathrm{C}$ for 4 minutes and under a compaction pressure of $35 \mathrm{MPa}$. Samples with dimensions $\varnothing 40 \times$ $15 \mathrm{~mm}$ were produced. The deformation samples used in the compression tests $(\varnothing 5 \times 8 \mathrm{~mm})$ were cut from the SPSed specimens using electrical discharge machining.

Compression testing. Compression tests were carried out in vacuum of $10^{-4} \mathrm{mbar}$. Nine specimens were heated to testing temperature with a rate of $10 \mathrm{~K} / \mathrm{s}$, soaked for $5 \mathrm{~min}$, and then upset under constant strain rate conditions to a strain of $\varepsilon=0.9$ (cf. Fig. 1). Nine different combinations of strain rate $(\dot{\varepsilon}=0.001,0.01,0.1 / \mathrm{s})$ and temperature $\left(T=1150,1200,1250^{\circ} \mathrm{C}\right)$ were tested. Each specimen was quenched immediately after upsetting. To determine the effect of forming on the microstructure and damage evolution, the compression samples were sectioned axially and prepared by means of standard metallographic techniques. The sample surfaces were treated by vibrational polishing. The sections were also analyzed using scanning electron microscopy. 


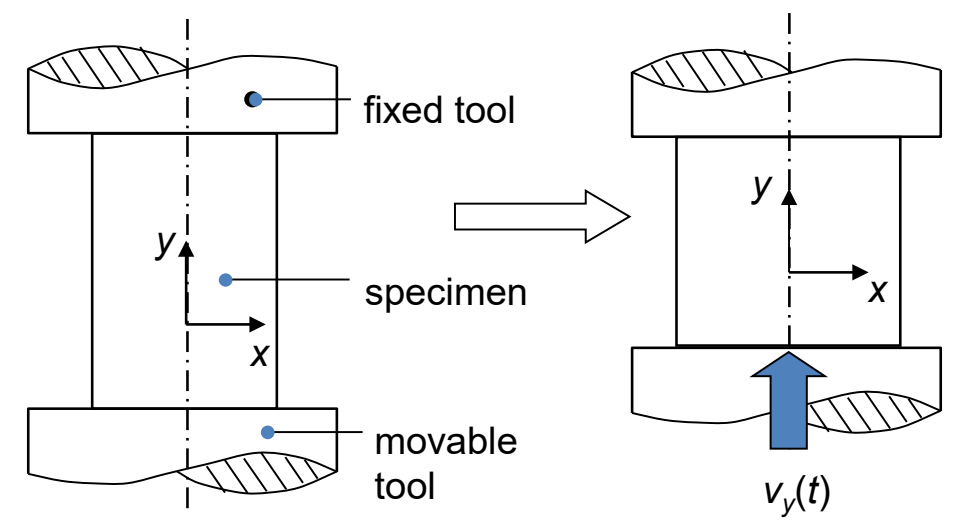

Fig. 1. Illustration of the compression test. $v_{y}(\mathrm{t})$ denotes the ram speed.

Finite element model. A finite element model was set up in ABAQUS/standard, in which a cylindrical specimen is axially compressed with friction, in accordance with the dilaotmeter upsetting tests. The simulation is performed as axisymmetric model. The initial mesh is rectangular and has 260 elements, i.e. 13 by 20 in $x$ - and $y$-direction. Axial symmetry and mirror symmetry are used to reduce the computational time. The initial specimen temperature is prescribed homogeneously inside the specimen according to the experimental conditions and evolves due to dissipation inside the workpiece and heat transfer. The material behaviour is elastic-viscoplastic with a flow stress model and damage model as discussed below.

Flow stress model. The constitutive equations relating the flow stress with temperature, strain rate and strain are calibrated from the data of the hot compression tests. Based on the experimental results, a phenomenological flow stress model for hot deformation of the present alloy was established. The subroutine UHARD is used to implement the model, which passes the flow stress, Eq. (9), and its derivatives with respect to strain $\varepsilon$, strain rate $\dot{\varepsilon}$ and temperature $T$ to the solver. All other model equations (1-8) define quantities used in the flow stress model. Eq. (8) describes the dynamic recrystallization (DRX) kinetics for the recrystallizing phases $i$ in the material. Due to the fact that the microstructure consists of multiple phases, the recrystallized grain sizes of the phases undergoing recrystallization are considered separately. Eqs. (3-7) detail the characteristic strains and stresses used to describe the dependence of flow stress on $Z$, the Zener-Hollomon parameter, Eq. (1), where $\dot{\varepsilon}$ is the strain rate, $Q_{w}$ is the apparent activation energy for hot deformation, $R$ the universal gas constant and $T$ the temperature in Kelvin. Eq. (2) details the strain hardening behavior of the phase $i$ in the unrecrystallized (0) and recrystallized (1) state. The empirical equation proposed by Cingara \& McQueen [8] is used here, where $\mathrm{C}$ is a constant which is weakly dependent on temperature and strain rate and determines the curvature of the flow curve up to the peak.

Table 1. Model equations for the TNB-V5 alloy. The model parameters are $Q_{w}, \alpha, a_{1}-a_{3}, e_{1}-e_{4}, f_{1}-f_{3}$, $h_{1}-h_{3}, k_{i}, q_{i}$ and constitute the parameter vector $\boldsymbol{\theta}$.

\begin{tabular}{|l|l|}
\hline Zener Hollomon Parameter & $Z=\dot{\varepsilon} \exp \left(\frac{Q_{w}}{R T}\right)$ \\
\hline Strain hardening & $\sigma_{0,1}=\sigma_{p}\left[\frac{\varepsilon}{\varepsilon_{p}} \exp \left(1-\frac{\varepsilon}{\varepsilon_{p}}\right)\right]^{C}$ \\
\hline Critical strain & $\varepsilon_{c r}=\alpha \varepsilon_{p}$ \\
\hline Peak strain & $\varepsilon_{p}=a_{1} \cdot d_{0}^{a_{2}} \cdot Z^{a_{3}}$ \\
\hline Steady state stress & $\varepsilon_{s s}=e_{1} \cdot \varepsilon_{m}+e_{2} \cdot d_{0}^{e_{3}} \cdot Z^{e_{4}}$ \\
\hline Peak stress & $\sinh \left(f_{3} \cdot \sigma_{p}\right)=f_{1} \cdot Z^{f_{2}}$ \\
\hline
\end{tabular}




\begin{tabular}{|l|c|}
\hline Steady state stress & $\sinh \left(h_{3} \cdot \sigma_{s s}\right)=h_{1} \cdot Z^{h_{2}}$ \\
\hline Softening kinetics & $X_{i}=1-\exp \left[k_{i} \cdot\left(\frac{\varepsilon-\varepsilon_{c r}}{\varepsilon_{s s}-\varepsilon_{c r}}\right)^{q_{i}}\right], \quad \varepsilon \geq \varepsilon_{c r}$ \\
\hline Flow stress & $\sigma_{Y}=\left\{\left(1-\sum_{i} X_{i}\right) \sigma_{0}+\sum_{i} X_{i} \sigma_{i} \quad\right.$ if $\varepsilon<\varepsilon_{c r}$ \\
&
\end{tabular}

Damage modeling. A model for damage evolution in hot working has been applied in the current study. The model is being developed in the collaborative research project Transregio 188, with steel as primary area of application. It allows to quantify the effect of the stress state on the nucleation of voids. Since this work focuses on deformation conditions that minimize damage, this model is employed here, assuming a dilute concentration of voids in the material. For forming under conditions with severe internal material damage, an extended approach is necessary, which accounts for the interaction of voids. The model is based on the evolution equation for the damage variable $D$, which represents the number of voids per material volume, put forward by Horstmeyer et al. [10]:

$$
\dot{D}=D \frac{\dot{\varepsilon} \sqrt{d}}{\mathrm{~K}_{\mathrm{IC}} f^{\frac{1}{3}}}\left(a\left(\frac{4}{27}-\frac{\mathrm{J}_{3}{ }^{2}}{\mathrm{~J}_{2}{ }^{3}}\right)+b \frac{\mathrm{J}_{3}}{\mathrm{~J}_{2}{ }^{\frac{3}{2}}}+c\left\|\frac{I_{1}}{\sqrt{\mathrm{J}_{2}}}\right\|\right)
$$

Coupling to microstructural features such a grain size $d$ is possible through the explicit dependence of the evolution of the damage variable $D$ on $\mathrm{d}$ as well as on the volume of potential failure sites, $f$. The dependence on stress state is taken into account via the term in the brackets, which takes into account the stress triaxiality by the ratio of the first invariant of the Cauchy stress tensor $\sigma, I_{1}=\sigma_{1}+\sigma_{2}+\sigma_{3}$, and the square root of the second invariant of the stress deviator, $J_{2}$. The other terms contain ratios of the third and second invariant of the stress deviator, $J_{3}$ and $J_{2}$, which account for the dependence of damage evolution on the Lode angle. $K_{I C}$ is the fracture toughness of the material, $a, b, c$ are model parameters.

The FE result in Figure 2 shows the damage variable calculated in the compression specimen. The largest amount of damage is predicted in the center of the specimen due to the high plastic strain and on the outer radius of the top (and bottom) face which is brought into contact with the dies during forming. The optimal control problem focuses on the damage in the center of the specimen, which is more critical than near surface regions due to the fact that any forged component will be machined to the final shape.

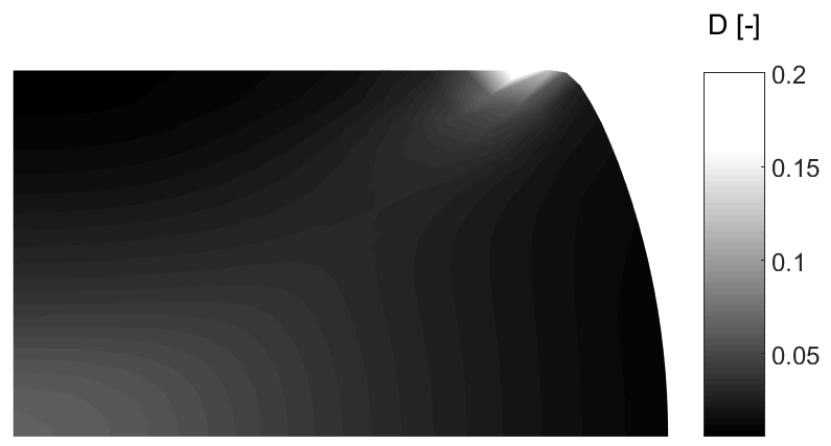

Fig. 2. Output of the damage model for a compression test at $1200{ }^{\circ} \mathrm{C}$ and a strain rate of $0.01 / \mathrm{s}$. Axial and mirror symmetry are used. 
Model parameter identification. The parameters $\boldsymbol{\theta}$ of the flow stress model as well as the damage model were determined by inverse methods, Eq. (11). For the calibration of the material model, all nine available flow curves were used and the optimization problem

$$
\left\{\begin{array}{l}
F(\theta)=\sum_{T_{i}} \sum_{\dot{\varepsilon}_{j}} \sum_{\varepsilon_{k}}\left(\sigma_{Y}^{(\text {exp })}\left(T_{i}, \dot{\varepsilon}_{j}, \varepsilon_{k}\right)-\sigma_{Y}^{(\operatorname{sim})}\left(T_{i}, \dot{\varepsilon}_{j}, \varepsilon_{k}, \theta\right)\right)^{2} \\
\theta^{*}=\arg \min F(\theta)
\end{array}\right.
$$

was solved using the Levenberg-Marquardt algorithm. The parameter identification of the damage model was based on the void fraction obtained from scanning electron microscopic evaluation of the specimens that showed damage. More details on parameter identification are reported in previous work [9].

Optimization of the punch speed. The control input of the process is the punch velocity $v_{y}(t)$, which determines the evolution of the variables responsible for damage evolution according to Eq. (10). With the plastic strain rate $\dot{\varepsilon}$ and the stress state $\sigma$ given by the Cauchy stress tensor in the specimen, the damage variable $D$ can be computed everywhere in the specimen. The problem can be stated as follows:

$$
\begin{aligned}
& \min _{v_{y}(t)} t_{1} \\
& \int_{0}^{t_{1}} v_{y}(t) d t=\Delta h=\text { const. } \\
& \max _{v_{y}(t)} D(t, \Omega)<D_{c r} \forall t \in\left[0 ; t_{1}\right] \text { and } \forall(x, y) \in \Omega
\end{aligned}
$$

The problem is hence to minimize the process time $t_{1}$, which is necessary to form the specimen to a height reduction of $\Delta h$, while keeping the damage variable $D$ smaller than a critical value at all times and in the entire workpiece, given by the domain $\Omega$. The critical value is the damage value obtained from upsetting at a constant ram speed, which should not be exceeded at variable speed.

The domain is restricted to the center of the specimen. In order to simplify the problem, the velocity function is represented in the following way: initially, a constant ram speed $(v=0.01$ $\mathrm{mm} / \mathrm{s}$ ) is used, which corresponds to a strain rate of $\dot{\varepsilon}=0.01 / \mathrm{s}$. This is the typical speed of deformation which can be imposed without observable damage in the specimen. At the time $t_{0}$, the punch speed is increased according to the following velocity profile

$$
v_{y}(t)=\left\{\begin{array}{cc}
v_{0} & t<t_{0} \\
v_{0}+\left(v_{1}-v_{0}\right) \xi^{3}\left(10-15 \xi+6 \xi^{2}\right) & t \geq t_{0}
\end{array}\right.
$$

with $\xi=\frac{t-t_{0}}{t_{1}-t_{0}}$. This is the velocity definition for the time span in which the ram accelerates. It is chosen for two reasons: (1) it defines a smooth, jerk-free acceleration of the ram; (2) it allows to speed up the ram after some initial time at constant speed, which may be needed to for initiating microstructural transformations such as recrystallization increasing the damage-tolerance of the micrcostructure.

The final time $t_{1}$ is computed from the velocity $v_{l}$ so that a constant height reduction is obtained. The variables $t_{0}$ and $v_{1}$ are hence used to determine a candidate for an optimized velocity profile. This velocity profile parametrizes the starting time for accelerating the punch as well as the final 
punch speed. This velocity profile can be defined as 'amplitude' in ABAQUS. The parameters defining the profile are set by the optimizer, which is implemented in MATLAB. The simulation results are processed using python scripts in ABAQUS and read into the optimizer.

\section{Results and Discussion}

In this section the experimental and simulation results are given, and the solution of the optimal control problem is validated.

Microstructure evaluation. The specimens which were tested at constant strain rate were sectioned and analyzed by SEM to reveal damage in the microstructure. The material produced by SPS was also investigated to analyze possible defects in the initial microstructure. The SEM analysis of the initial state shows a dense, defect-free microstructure consisting of three phases, $\alpha_{2}$, $\beta_{0}$ and $\gamma$. After compression testing, damage in the form of voids is found in the specimens deformed under unstable conditions. These conditions can be visualized as dark gray region in the processing map, cf. Fig. 3.

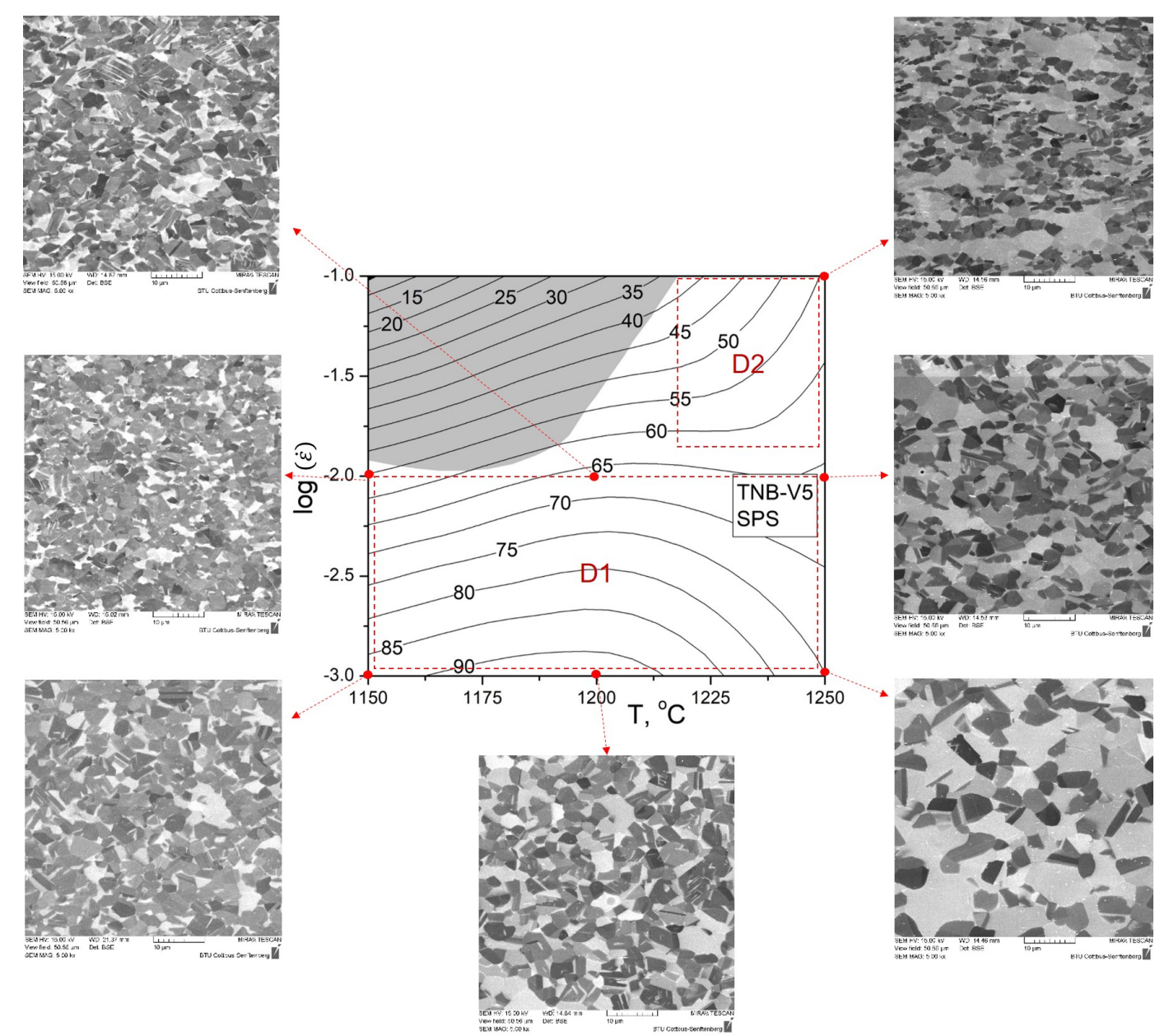

Fig. 3. Processing map for the TNB-V5 alloy showing conditions for damage-free forming and void formation at a strain of $\varepsilon=0.5$. D1 and D2 are safe regions showing superplasticity and dynamic recrystallization, respectively.

Optimization results. The optimization process was run using the ABAQUS model coupled with the MATLAB optimizer. It stops at the solution $t_{0}=0.05 \mathrm{~s}$ and $v_{l}=0.135 \mathrm{~mm} / \mathrm{s}$ after 79 function evaluations, i.e. finite element computations. Using these settings, the process can be accelerated by $\sim 25 \%$, i.e. the forming process needs only $75 \%$ of the original process time. The damage constraint only mildly depends on $t_{0}$, i.e. the point in time where the punch accelerates, so that this parameter could be omitted and the punch can be accelerated right from the start of the process. 
Validation. The velocity profile found from the optimization was used to perform a validation experiment at $1200{ }^{\circ} \mathrm{C}$ in which the velocity profile found by optimization was imposed on the cylindrical specimen in the upsetting test. Fig. 4 shows stress-strain curves obtained with the deformation dilatometer, which represents the true stress and strain up to the initiation of bulging. The SEM micrographs of the deformed specimen shows no damage in both cases, constant and variable strain rate.
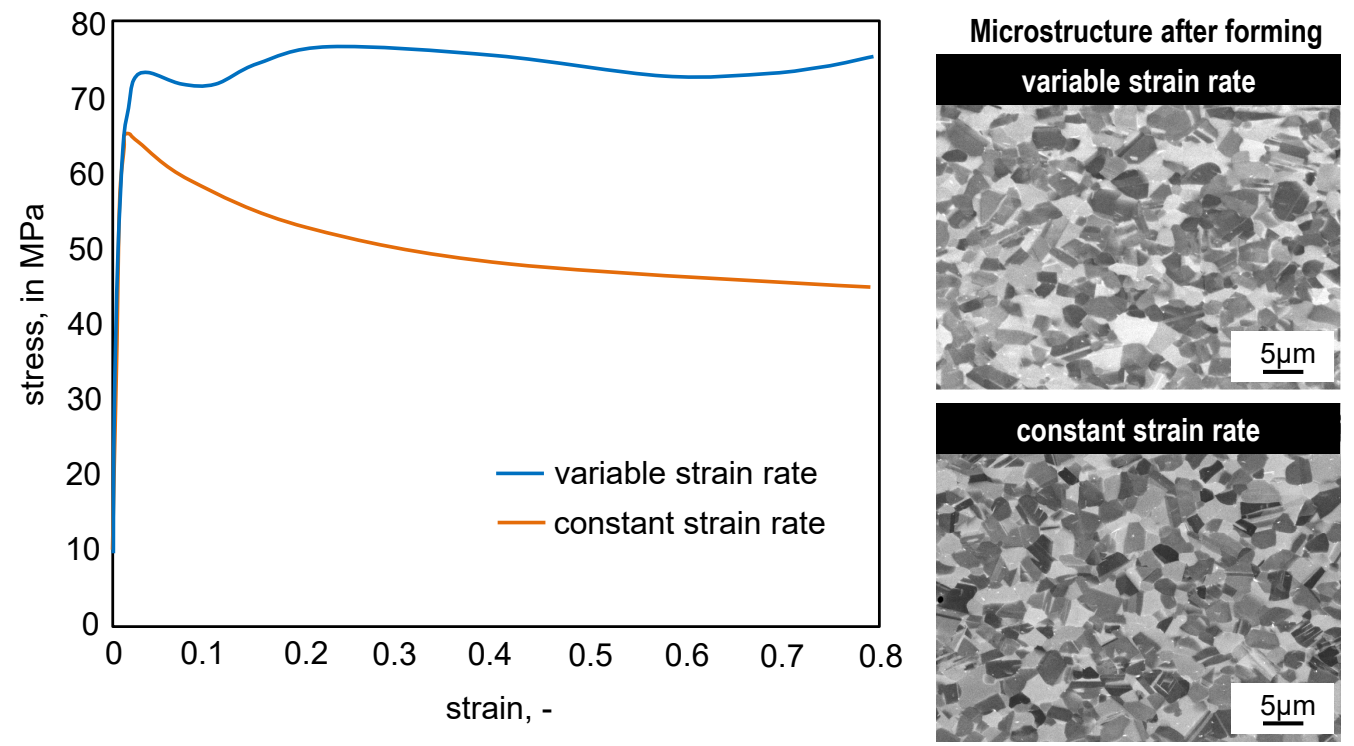

Fig. 4. Result of the validation experiment.

Discussion. It is remarkable that the optimizer found feasible parameters for a given velocity profile that led to deformation below a stress level slightly exceeding the peak stress that is observed at a constant strain rate of $\dot{\varepsilon}=0.01 / \mathrm{s}$. The acceleration of the punch thus seems to compensates for the softening, keeping the material roughly below a certain stress level. It can be concluded that the acceleration of the process was successful, albeit for a quite simple compression test. The variation of stress triaxiality in the specimen is small, so that it is reasonable to keep the specimen at constant stress level. In a real forged component, more complex stress states will arise, and it may become more difficult to accelerate the process, since the material volume with an unfavourable stress state, possibly in combination with a lower temperature, will determine the maximum forming speed.

\section{Summary}

In this work, possibilities for speeding up isothermal forging by increasing the punch velocity during cylinridcal compression were analyzed. The following conclusions can be drawn:

- For compression testing with rough plates, inhomogeneous deformation occurs. The specimen can be sucessfully formed with a velocity profile that keeps the macroscopic (average) stress below a certain stress level.

- No damage was observed in the specimens after forming at variable speed.

- The velocity can be ramped up to almost twice the initial speed, yielding a time saving of $25 \%$.

- The optimization problem was solved by searching the optimal parameters for a prescribed velocity profile for the entire duration of the process.

Future work will focus on solving the optimization problem over a limited time span, thus moving from optimal control to model predictive control with the possibility to implement the optimization problem on the machine controller. 


\section{Acknowledgements}

The authors thank the German Research Foundation for funding obtained within the SFB Transregio 188 (Damage Controlled Metal Forming Processes - INST 212/392-1) through project C03, in which the damage model used in this work was developed, as well as for funding of the project BA4253/4-1 "Prozessbeschleunigung des Isothermschmiedens von Titanaluminiden durch mikrostrukturadaptive Geschwindigkeitssteuerung".

\section{References}

[1] N.E. Paton, J.C. Williams, Deformation of body-centered-cubic titanium-vanadium single crystals, in Proc. of the Second International Conference on the Strength of Metals and Alloys, Pacific Grove, California, 30 Aug. - 4 Sept. 1970. Vol. I. Metals Park, Ohio, 1970, pp 108-112.

[2] D. Hautmann, Titanaluminid - Das beste aus zwei Welten, MTU Report 01-13 (2013) 25-29.

[3] P. Janschek, Forging Titaniumaluminide Parts, Materials Science Forum 638 (2010).

[4] F. Kong, N. Cui, Y. Chen, X. Wang, N. Xiong, Characterization of hot deformation behavior of as-forged TiAl alloy, Intermetallics 55 (2014) 66-72.

[5] B. Liu, Y. Liu, W. Zhang, J.S. Huang, Hot deformation behavior of TiAl alloys prepared by blended elemental powders, Intermetallics 19:2 (2011) 154-159.

[6] M. Bambach, I. Sizova, A. Sviridov, J.A. Stendal, M. Günther, Batch Processing in Preassembled Die Sets-A New Process Design for Isothermal Forging of Titanium Aluminides. Journal of Manufacturing and Materials Processing, 2:1 (2018) 1.

[7] P. Janschek, Wrought TiAl Blades, Materials Today: Proceedings 2 (2015) 92-97.

[8] A. Cingara, H.J. McQueen, New formula for calculating flow curves from high temperature constitutive data for 300 austenitic steels, J. Mater. Proc. Tech. 36 (1992) 31-42.

[9] M. Bambach, I. Sizova, S. Bolz, S. Weiß, Devising Strain Hardening Models Using KocksMecking Plots-A Comparison of Model Development for Titanium Aluminides and Case Hardening Steel. Metals, 6:9 (2016) 204.

[10]M.F. Horstemeyer, A.M. Gokhale, A void-crack nucleation model for ductile metals. International Journal of Solids and Structures, 36:33 (1999) 5029-5055. 